\title{
Randomised clinical trial: the DPP-4 inhibitor, vildagliptin, inhibits gastric accommodation and increases glucagon-like peptide-1 plasma levels in healthy volunteers
}

\author{
Alessandra Rotondo $^{1}$ | Imke Masuy $^{1}$ (D) | ${\text { Wout } \text { Verbeure }^{1} \text { | Jessica R. Biesiekierski }}^{1,2}$ | \\ Eveline Deloose $^{1}$ (D) | Jan Tack ${ }^{1}$
}

${ }^{1}$ Translational Research Center for Gastrointestinal Disorders, KU Leuven, Leuven, Belgium

${ }^{2}$ Department of Rehabilitation, Nutrition and Sport, La Trobe University, Melbourne,

Vic., Australia

Correspondence

Prof. Jan Tack, TARGID, University of

Leuven, Leuven, Belgium.

Email: jan.tack@kuleuven.be

Funding information

Supported by University of Leuven (Methusalem grant) and by the Flanders Research Foundation (FWO). JRB and ED are postdoctoral fellows of the FWO.

\section{Summary}

Background: Dipeptidyl peptidase-4 (DPP-4) inactivates glucagon-like peptide-1 (GLP-1). Whether DPP-4 inhibition affects GLP-1 metabolism and/or food intake in humans remains unknown.

Aims: To evaluate the effect of vildagliptin (DPP-4 inhibitor) on gastric accommodation and ad libitum food intake in healthy volunteers (HVs)

Methods: The effects of acute oral vildagliptin administration (50 mg) were evaluated in two randomised, placebo-controlled, single-blinded trials. Protocol 1 ( $n=10$, $32.3 \pm 3$ years, $23.4 \pm 0.7 \mathrm{~kg} / \mathrm{m}^{2}$ ): 60 min after treatment, a nutrient drink (270 kcal) was infused intragastrically and intragastric pressure (IGP) was measured for $1 \mathrm{~h}$. Protocol $2\left(\mathrm{n}=10,24.3 \pm 0.8\right.$ years, $\left.22.3 \pm 0.9 \mathrm{~kg} / \mathrm{m}^{2}\right): 60 \mathrm{~min}$ after treatment, HVs consumed one nutrient drink (300 kcal). Thirty minutes thereafter, HVs ate ad libitum from a free-choice buffet for $30 \mathrm{~min}$. Blood was collected at several time points to measure active GLP-1 plasma levels.

Results: During the first 20 min after nutrient infusion, the drop in IGP was smaller after vildagliptin compared to placebo (treatment-by-time interaction effect: $P=0.008)$. No differences were seen on epigastric symptom scores. Planned contrast analysis showed that active GLP-1 levels were higher after vildagliptin compared to placebo $(P=0.018)$ only after nutrient ingestion. Total food intake $(316.38 \pm 58.89 \mathrm{~g}$ vs $399.58 \pm 63.02 \mathrm{~g}, \quad P=0.359)$ and total caloric intake $(594.77 \pm 115.17 \mathrm{kcal}$ vs $742.77 \pm 107.10 \mathrm{kcal}, P=0.371)$ did not differ between treatments.

Conclusions: Vildagliptin inhibits gastric accommodation without affecting epigastric symptom scoring in HVs. Active GLP-1 plasma levels were increased after vildagliptin treatment, but the increase was not sufficient to affect ad libitum food intake. The study was registered at Clincialtrials.gov (NCT 03500900). 


\section{1 | INTRODUCTION}

Dipeptidyl peptidase-4 (DPP-4) is a complex enzyme expressed in epithelial cells, capillary endothelia and lymphocytes of the gastrointestinal tract, kidney, liver and brain. ${ }^{1}$ It is a ubiquitous serine protease responsible for the degradation of several endogenous peptides including the L-cell-derived peptide glucagon-like peptide-1 (GLP-1). ${ }^{2}$ DPP-4 transforms the active form of GLP-1 (GLP-1(7-36)) to an inactive form (GLP-1(9-36)) by removing the N-terminal dipeptide, making it incapable of activating its receptor. ${ }^{3}$

The biological actions of active GLP-1 converge at multiple levels on the modulation of gastrointestinal motility and food intake. GLP-1 is an incretin, which stimulates postprandial insulin synthesis and secretion and inhibits glucagon release. ${ }^{4}$ It is also known as an anorexigenic peptide that increases satiety. ${ }^{5,6}$ Additionally, GLP-1 acts as a mediator of the ileal brake, ${ }^{7}$ reduces gut motility, ${ }^{8,9}$ increases gastric accommodation, ${ }^{10,11}$ slows gastric emptying ${ }^{12}$ and inhibits gastric acid secretion. ${ }^{6,9,13,14}$

During the last decade, DPP-4 inhibitors have been developed to increase circulating concentrations of intact, biologically active peptides and they have been approved as an additional therapeutic approach for the treatment of type 2 diabetes mellitus (T2DM). Although there is no conclusive evidence that DPP-4 has a major role in GLP-1 metabolism in vivo, it is conceivable that one of the mechanisms by which DPP-4 inhibition leads to hyperinsulinaemia is by protecting GLP-1 from degradation. ${ }^{15,16}$

Among the DPP-4 inhibitors, vildagliptin (Galvus ${ }^{\circledR}$; Novartis, Basel, Switzerland) has been approved for the treatment of T2DM in Europe. A dose of $50 \mathrm{mg}$ has been shown to provide a potent inhibition of DPP-4, starting 45 min after meal ingestion, reaching its maximum plasma concentration within $1-2 \mathrm{~h}$ and lasting for more than $12 \mathrm{~h} .^{17}$ Chronic use of vildagliptin has been shown to have neutral effects on body weight and plasma lipid profiles in T2DM. ${ }^{18}$ The effects of vildagliptin on food intake and gastrointestinal motility in man, and the possible involvement of increased GLP-1 plasma halflife are incompletely elucidated.

Gastric accommodation is a major determinant of food intake and nutrient volume tolerance. ${ }^{19}$ It is a reflex response that enhances the storage capacity of the stomach during food intake through a decrease of the muscle tone in the proximal stomach. This mechanism increases gastric compliance while keeping IGP low. ${ }^{20}$ We recently described a novel technique to measure gastric accommodation during intragastric nutrient drink infusion in humans based on IGP measurements. ${ }^{19}$ IGP is determined by the gastric muscle tone and we have demonstrated that during intragastric nutrient infusion, the IGP decreases initially to gradually increase thereafter. The drop in IGP requires nitric oxide synthase activity and reflects the gastric accommodation reflex. Moreover, we have shown that the IGP recovery from nadir (lowest IGP) is a determinant of satiation. $^{19}$

In a mechanistic study in T2DM using single-photon emission computed tomography (SPECT), no significant changes in gastric volume and meal-induced satiation were found after an overnight fast following 10 days of vildagliptin treatment. Fasting GLP-1 levels were not altered; however, postprandial GLP-1 levels were significantly higher following vildagliptin treatment. ${ }^{21}$ It is unclear whether assessment of nutrient tolerance and satiation would differ if this was done during vildagliptin-induced rises in GLP-1 plasma levels.

Hence, the aim of the present study was to examine whether acute administration of the DPP-4 inhibitor vildagliptin would affect (a) gastric accommodation, evaluated as changes in IGP during a meal, and (b) food intake, at the moment of vildagliptin-induced rises in concentrations of GLP-1.

\section{MATERIALS AND METHODS}

\section{1 | Overall design and participants}

The present study consisted of two randomised, placebo-controlled, single-blinded, crossover trials to evaluate the effect of acute administration of vildagliptin on (a) IGP during intragastric nutrient infusion and on (b) active GLP-1 plasma levels and ad libitum food intake in healthy volunteers (HVs). Ten HVs participated in each protocol. They were recruited from a database of volunteers who previously participated in studies within our research group. Exclusion criteria included the presence of symptoms or a history of gastrointestinal diseases, diabetes, drug allergies, psychological disorders and major gastrointestinal surgery. Subjects came to the research facility (University Hospital, Leuven, Belgium) after at least $6 \mathrm{~h}$ of fasting. They were asked to refrain from alcohol, tea and coffee at least $12 \mathrm{~h}$ before participation and from smoking cigarettes at least $1 \mathrm{~h}$ before the start of the experiment. The study was approved by the Medical Ethics Committee of the University Hospitals Leuven and was performed in accordance with the declaration of Helsinki and the BMJ guidelines. All participants gave written informed consent before any study procedures were performed. The study was registered at Clincialtrials.gov as NCT03500900.

\subsection{Treatment and randomisation}

In both study protocols, vildagliptin (Galvus ${ }^{\circledR}$, Novartis; $50 \mathrm{mg}$ ) was administered orally as a tablet 20 min after the start of each experiment. This dosage has been approved for the treatment of T2DM in Europe. ${ }^{21,22}$ A matching placebo tablet was administered in the placebo arm of each protocol. Participants received both treatments (vildagliptin and placebo) in a randomised and counterbalanced order. The treatment randomisation code was automatically generated by a computer program. A washout period of at least 1 week separated both treatments arms.

\section{3 | Study protocols}

The study consisted of two independent protocols to evaluate gastric accommodation and ad libitum food intake. 


\subsection{1 | Protocol 1: IGP measurement during infusion of a liquid meal}

IGP was assessed using a high-resolution solid-state manometry system (HRM) with 36 channels spaced at $1 \mathrm{~cm}$ intervals (Manoscan 360; Sierra Scientific Instruments, Los Angeles, CA, USA; Manoview analysis software v2.0.1). Gastric accommodation was evaluated by measuring changes in IGP during an intragastrically infused liquid meal. Upon arrival at the research facility, after a fasting period of at least $6 \mathrm{~h}$, the manometry catheter was positioned through the nose into the stomach so that at least one channel was located at the lower oesophageal sphincter (LES; detected as a clearly elevated pressure zone compared to oral and aboral areas). IGP of the proximal stomach was measured using the average pressure of the first five channels that were clearly positioned below the LES or the pressure area influenced by the LES (approximately $3-8 \mathrm{~cm}$ under the LES as determined from the high-resolution plot). ${ }^{19}$ In addition, an infusion catheter (Flocare; Nutricia, Bornem, Belgium) was positioned in the proximal stomach through the mouth. The tip of the infusion catheter was positioned approximately $5 \mathrm{~cm}$ below the LES. The position of both catheters was verified using fluoroscopy. Thereafter, the catheters were fixed with adhesive tape to avoid replacement and the participant was asked to lie on a bed in a semi-recumbant position and stay there for the remainder of the experiment.

After a stabilisation period of 20 min, study medication was administered orally in a single-blinded fashion. One hour hereafter, a nutrient drink (Nutridrink, Nutricia; 150 kcal, 6 g protein, $18.4 \mathrm{~g}$ carbohydrate, and $5.8 \mathrm{~g}$ lipid/100 mL) was intragastrically infused at a constant speed of $60 \mathrm{~mL} / \mathrm{min}$ (determined by an automated system using a peristaltic pump) for $3 \mathrm{~min}$ ( $270 \mathrm{kcal}$ in total). Sixty minutes after infusion, the catheters were disconnected and removed from the participant. Epigastric sensations and symptoms (hunger, appetite, fullness, nausea, bloating, belching, epigastric pain and abdominal cramps) were scored every 5 min throughout the study on a $10 \mathrm{~cm}$ visual analogue scale (VAS). See Figure 1 for the protocol outline.

\subsection{2 | Protocol 2: Ad libitum food intake after a pre-meal load}

Participants came to the research facility after a fasting period of at least $6 \mathrm{~h}$. An intravenous catheter was placed in an antecubital vein to collect blood samples in order to measure the plasma concentration of active GLP-1 at different time points. Thereafter, study medication was orally administered. One hour after dosing, subjects consumed $200 \mathrm{~mL}$ (300 kcal) of nutrient drink (Nutridrink, Nutricia). Thirty minutes later, an excess free-choice buffet was served. ${ }^{17}$ The buffet has been previously described. ${ }^{23}$ Briefly, it included a variety of pre-sliced ready-to-eat food items, including bread, ham, cheese, lettuce, tomato, mayonnaise, jam, sweets, crisps, rice pudding, waffles, chocolate, apples and bananas. All food items were weighed pre- and postprandially to calculate total food and energy intake. The total caloric value of the buffet meal was $2330 \mathrm{kcal}$. It contained

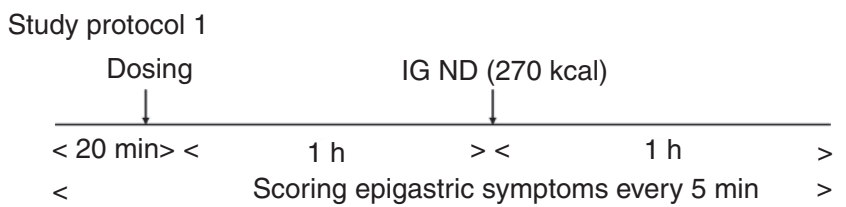

Study protocol 2

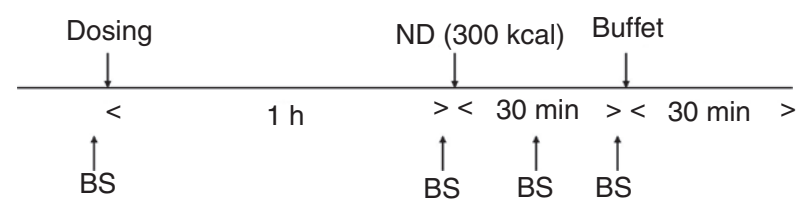

FIGURE 1 Schematic overview of the protocol outline. Both protocols were single-blinded, randomised, placebo-controlled, crossover trials. Either placebo or $50 \mathrm{mg}$ vildagliptin was administered orally (dosing). Intragastric pressure was measured continuously in protocol 1 with the use of high-resolution manometry. Epigastric symptoms (hunger, appetite, fullness, nausea, bloating, belching, epigastric pain and abdominal cramps) were scored every 5 min on 10-cm visual analog scales with endpoints. Nutridrink was administered intragastrically in the first protocol through a nasogastric feeding tube, placed with its tip in the proximal part of the stomach. Blood samples (BS) were collected at regular time points during the second protocol to measure active GLP-1 plasma concentrations. Nutridrink was ingested orally 30 min prior to an excess-choice buffet of $2330 \mathrm{kcal}$. Subjects were instructed to eat until maximum satiation or for a duration of $30 \mathrm{~min}$. Hunger and satiation were scored at the start and end of the buffet meal on visual analog scales

$55 \mathrm{~g}$ protein, $94 \mathrm{~g}$ fat (of which $32 \mathrm{~g}$ was saturated) and $291 \mathrm{~g}$ carbohydrates. Subjects were instructed to eat ad libitum until maximum satiation or for a maximum of $30 \mathrm{~min}$. Hunger and satiation were scored on a $10 \mathrm{~cm}$ VAS before and after the buffet. Blood samples to assess active GLP-1 plasma levels were collected immediately before drug or placebo administration, $5 \mathrm{~min}$ before and 15 min after intake of the nutrient drink and immediately before the buffet (30 min after intake of the nutrient drink). Samples were collected in $10 \mathrm{~mL}$ EDTA tubes (Becton Dickinson and Company, Franklin Lakes, NJ, USA) containing $50 \mu \mathrm{L}$ aprotinin per $\mathrm{mL}$ blood (500 kIU aprotinin/mL; Merck Millipore, Darmstadt, Germany) and DPP-4 inhibitor (10 $\mu \mathrm{L} / \mathrm{mL}$, Merck Millipore). All samples were stored on ice until centrifugation at $4^{\circ} \mathrm{C}$ for $10 \mathrm{~min}$ at $3000 \mathrm{~g}$, after which plasma was separated and stored at $-80^{\circ} \mathrm{C}$ until analysis. Active GLP-1 was measured using a Multi-Array Active GLP-1 Assay Kit (K150JWC; Meso Scale Diagnostics, Gaithersburg, MD, USA). The lowest limit of detection was $0.12 \mathrm{pg} / \mathrm{mL}$. The intra-assay coefficient of variation was $3 \%$. See Figure 1 for the protocol outline.

\subsection{Outcomes}

The primary outcome of the first study protocol was to compare changes in IGP for $2 \mathrm{~h}$ after administration. The secondary outcome was to evaluate changes in epigastric symptom scores for $2 \mathrm{~h}$ after administration. The primary outcome of the second study protocol was to compare ad libitum food intake $30 \mathrm{~min}$ after placebo and 
vildagliptin treatment measured as both total weight and total caloric intake. The secondary outcome was to compare plasma levels of active GLP-1 levels between the two treatment arms for $2 \mathrm{~h}$ after administration.

\section{5 | Data and statistical analysis}

The study was designed to have an $80 \%$ power and two-sized $\alpha$ value of $5 \%$ to detect $30 \%$ differences in IGP drop during the nutrient challenge test. Data were analysed in Statistical Analysis Software (SAS). Normality of the data was evaluated using Shapiro-Wilk tests. Data are represented as mean \pm SEM.

\subsection{1 | IGP measurement}

The original data were imported from the recording software into Microsoft Excel (Microsoft, Redmond, WA, USA). To avoid influence from movement and artefacts caused by coughing, sneezing or swallowing, a moving median was calculated per channel from the original data (median value over 1 min of original data). Per channel, a baseline value was calculated from the moving median data as the average pressure during the last $5 \mathrm{~min}$ before the intragastric nutrient drink infusion.

The time course of IGP data was analysed in two parts: (a) from administration of study medication until $1 \mathrm{~h}$ after dosing, further referred to as fasted state; (b) from start of nutrient drink infusion until the end of the measurement, further referred to as fed state. Data were analysed as change from baseline using linear mixed models. To analyse IGP in the fed state, the average IGP measured during fasting was added as an independent covariate in the model to account for possible differences in IGP in the fasted state. Furthermore, treatment (within-subject) and time (within-subject) were the categorical independent variables included in the model. The effects of interest included the main effect of treatment (testing differences in IGP response between both treatments over all time points) and the treatment-by-time two-way interaction effect (testing differences in the time course of the IGP response between both treatments). The variance-covariance structure providing the best fit was chosen based on the minimum value of Akaike's information criterion (AIC). Planned contrast analysis was performed to look at differences in IGP per time block of 5 min. A step-down Bonferroni correction for multiple testing was performed for every planned contrast analysis.

\subsection{2 | Epigastric symptoms}

The time courses of the epigastric symptom scores (hunger, appetite, fullness, nausea, bloating, belching, epigastric pain and abdominal cramps) were analysed using linear mixed models. Treatment (withinsubject) and time (within-subject) were the categorical independent variables included in the model. The effects of interest included the main effect of treatment and the treatment-by-time two-way interaction effect. The variance-covariance structure providing the best fit was chosen based on the minimum value of AIC.

\subsection{3 | Active GLP-1 assay}

Data were log-transformed in order to obtain normal data distribution and analysed using linear mixed models. Treatment (within-subject) and time (within-subject) were the categorical independent variables included in the model. The effects of interest included the main effect of treatment and the treatment-by-time two-way interaction effect. Planned contrast analysis was performed to look at differences in active GLP-1 plasma concentrations per time point (before drug, before nutrient drink, after nutrient drink, before buffet). A step-down Bonferroni correction for multiple testing was performed for every planned contrast analysis.

\subsubsection{Food intake}

Food intake was compared between placebo and vildagliptin with the use of a paired Student's t-test for both weight and caloric intake.

\section{RESULTS}

Ten HVs were included in study protocol 1 . Their mean age was $32 \pm 3$ years, with a mean BMI of $23 \pm 1 \mathrm{~kg} / \mathrm{m}^{2}$. Ten HVs were included in study protocol 2 . Their mean age was $24 \pm 1$ years, with a mean BMI of $22 \pm 1 \mathrm{~kg} / \mathrm{m}^{2}$. For both protocols, half of the participants were male. All randomised subjects finished their study visits and analysis was performed on data of all subjects. None of the subjects reported any adverse events.

\subsection{Gastric accommodation is diminished after vildagliptin treatment}

Although vildagliptin was associated with a lower IGP in the fasted state, this difference was not significant (treatment main effect: $F=1.23, P=0.28$ ). There was a significant time and treatment-bytime two-way interaction effect $(F=1.65, P=0.001$ and $F=1.43$, $P=0.015$ respectively), indicating that IGP changes over time (over both treatments) and that the time course of the IGP differed significantly between vildagliptin and placebo. This significance is driven by the greater decrease in IGP after vildagliptin treatment compared to placebo. However, planned contrast analysis did not show significant differences between vildagliptin and placebo within any single 5 min time block ( $P>0.07$ for all; Figure 2).

During the intragastric infusion of nutrient drink (fed state), IGP decreased initially and gradually increased thereafter in both treatment arms (Figure 2). There was no significant difference in IGP between both treatments over all time points (treatment main effect: $F=3.08, P=0.097)$. However, the time course of IGP differed significantly between both treatments (time main effect: $F=2.44$, $P<0.001$; treatment-by-time interaction effect: $F=1.50, P=0.008$ ), with a reduced drop in IGP after vildagliptin treatment compared to placebo (Figure 2). Post hoc analyses revealed a significant difference 


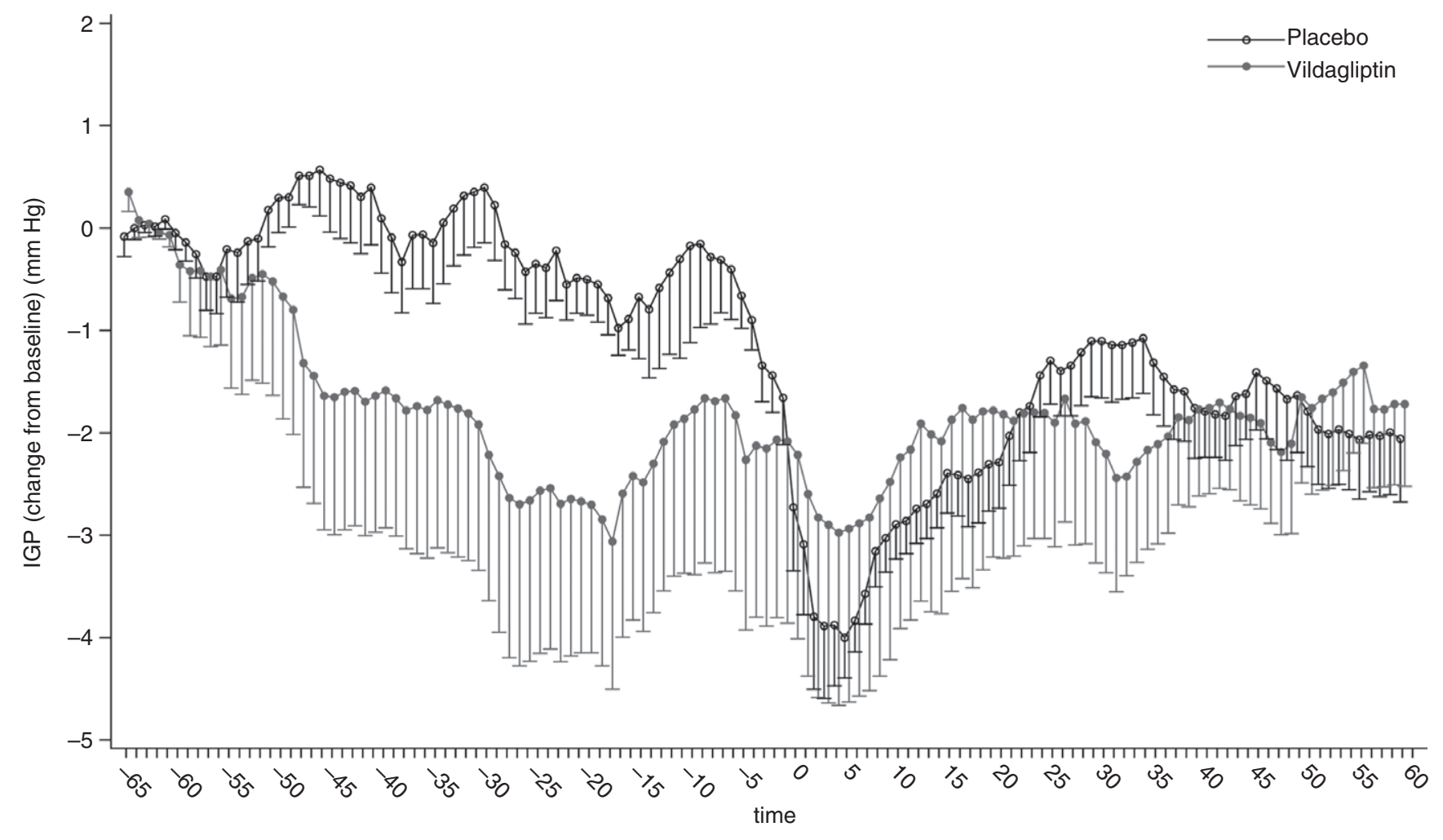

FIGURE 2 Intragastric pressure (IGP) changes from baseline in fasted state (minute -65 until minute 0 ) and fed state (from minute 0 until minute 60). Study medication was administered at $T=-60$. Infusion of the nutrient drink started at $T=0$ and ended at $T=3$. No significant differences were found in the fasted state $(P=0.28)$. During the intragastric infusion of nutrient drink, a reduction in IGP drop was observed following vildagliptin administration compared to placebo $(P=0.008)$. This difference was significant from the start of the nutrient drink infusion until $19 \mathrm{~min}$ after the start of the infusion ( $P=0.038$ for all $5 \mathrm{~min}$ time blocks)

between vildagliptin and placebo from the start of the nutrient drink infusion until 19 min after the start of the infusion $(P=0.038$ for all 5 min time blocks).

Vildagliptin did not significantly influence any epigastric symptom scores (treatment main effect: $P>0.09$ for all, treatment-by-time interaction effect: $P>0.57$ for all) (data not shown).

\subsection{Vildagliptin increases the plasma concentration of active GLP-1, but has no effect on food intake}

Active GLP-1 plasma concentrations changed significantly over time (time main effect: $F=34.14, P<0.001$ ). However, there was no significant difference in GLP-1 plasma concentration between both treatments (treatment main effect: $P=0.112$; treatment-by-time interaction effect: $F=1.47, P=0.251$ ). Planned contrast analysis showed that only after nutrient drink ingestion, the active GLP-1 plasma concentration was significantly higher for vildagliptin than placebo ( $P=0.018$ ), whereas there were no significant differences in active GLP-1 plasma concentration between vildagliptin and placebo before dosing, before nutrient drink ingestion or before the buffet ( $P>0.16$ for all; Figure 3 ).

Total food intake in weight was numerically lower after vildagliptin $(316.38 \pm 58.89 \mathrm{~g})$ compared to placebo $(399.58 \pm 63.02 \mathrm{~g})$, but this difference was not significant $(P=0.359$; Figure $4 A)$. Total energy content did also not differ significantly between placebo $(742.77 \pm 107.10 \mathrm{kcal})$ and vildagliptin $(594.77 \pm 115.17 \mathrm{kcal}$, $P=0.371$ ) (Figure 4B). Hunger and satiation were significantly different after the buffet compared to before the buffet in both treatment arms $(P<0.001$ for all). However, these scores were not affected by the treatment $(P>0.26$ for all, data not shown).

\section{DISCUSSION}

This study shows for the first time that the DPP-4 inhibitor, vildagliptin, is able to inhibit gastric accommodation. Gastric accommodation is a reflex response that leads to gastric relaxation to enhance the storage capacity of the stomach during food intake while keeping IGP low. Our group previously showed that gastric accommodation can be assessed by measuring IGP during infusion of a liquid meal and this technique has been validated by showing substantial concordance in results obtained with gastric barostat and IGP measurements in pharmacological studies. ${ }^{19,24,25}$ However, it is important to bear in mind that the measurement of IGP is an indirect measurement of gastric tone, and that several factors including gastric accommodation, gastric emptying and gastric content can influence IGP profiles over time. Furthermore, this technique does not 


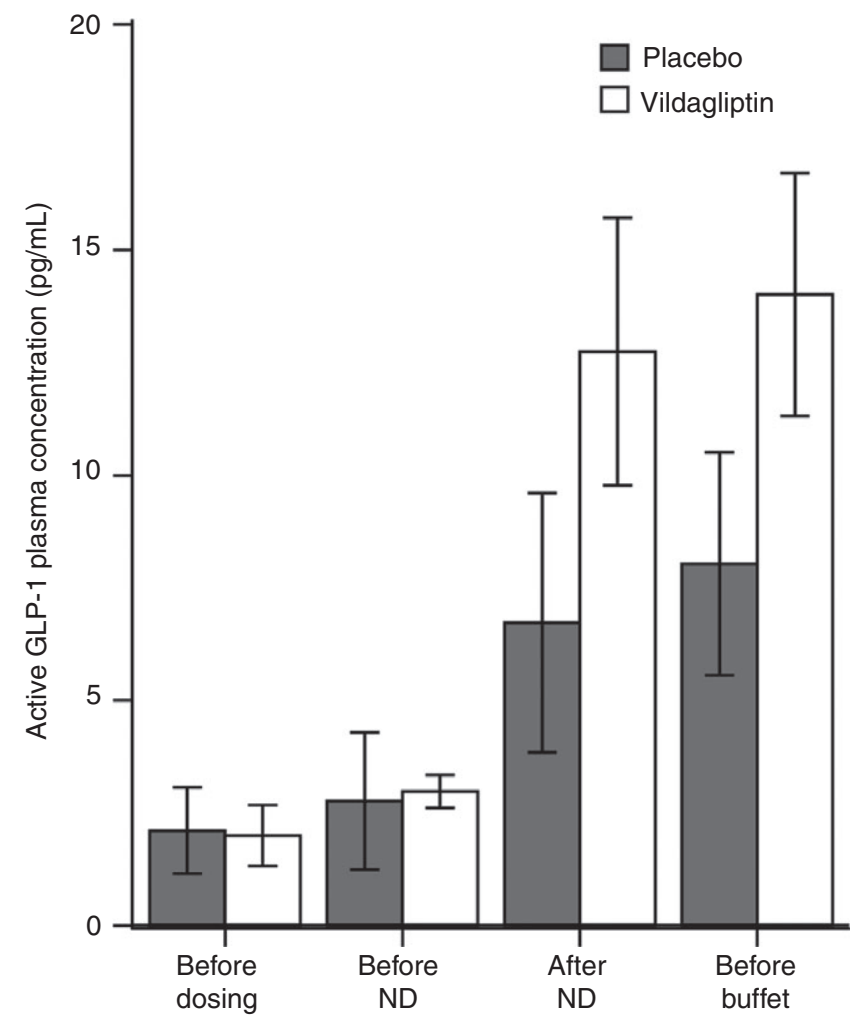

FIGURE 3 Active GLP-1 plasma concentrations before study drug administration, before and immediately after intragastric nutrient drink infusion and before buffet. Active GLP-1 plasma concentrations remained stable in the fasted state and increased after ingestion of a nutrient drink. Immediately after nutrient drink ingestion, the active GLP-1 plasma concentration was significantly higher for vildagliptin than placebo $(P=0.018)$. There were no significant differences in active GLP-1 plasma concentration between vildagliptin and placebo before dosing, before nutrient drink ingestion or before the buffet $(P>0.16$ for all)

serve to measure changes in gastric volume or distribution of the meal, in contrast to advanced imaging techniques such as magnetic resonance imaging, SPECT or scintigraphy. Nevertheless, this new technique represents a minimally invasive and more physiological alternative to barostat for the assessment of gastric accommodation, ${ }^{19,24,25}$ and shows that upon liquid meal infusion, IGP decreases initially to gradually recover thereafter. We have also demonstrated that the increase in IGP following the IGP drop is closely correlated with increasing satiation during nutrient drink infusion, suggesting that the IGP recovery during a meal is a determinant of satiation. ${ }^{19,24,25}$

In our experimental protocol, we investigated the effects of vildagliptin on IGP in fasted and in fed state. In the fasted state, IGP was numerically decreased after the intake of vildagliptin compared to placebo, but this difference was not significant due to large interindividual variability.

To assess the effects of vildagliptin on IGP in fed state, a fixed volume of a nutrient drink ( $270 \mathrm{kcal}$ ) was intragastrically infused $1 \mathrm{~h}$ after study drug administration (vildagliptin or placebo) in order to verify the effect of DPP-4 inhibition on gastric accommodation. Our results show that an acute dose of vildagliptin $(50 \mathrm{mg})$ inhibits the drop in IGP during nutrient drink administration, representing an inhibition of gastric accommodation. However, no differences were observed in hunger or satiation scores. It is conceivable that the effects of acute dose of vildagliptin on gastric tone are not strong enough to affect hunger and satiation. Alternatively, it is possible that (acute) inhibition of DPP-4 does not lead to an increase in GLP1 levels that is strong enough to exert an effect on hunger and satiation scores. The observed reduction of the IGP drop is in line with the results of a previous study of our group, where an acute dose of liraglutide, a GLP-1 analogue, also inhibited gastric accommodation. ${ }^{24}$ However, other studies using (99 m)Tc SPECT imaging showed contradicting results, namely an increase in gastric volumes after treatment with a GLP-1 agonist ${ }^{11,26}$ or a decreased gastric accommodation after acute treatment with a GLP-1 receptor antagonist. ${ }^{27}$ We only found an increase in gastric volumes, represented by a lower IGP, in the fasted state, although the difference with placebo was not significant. These differences could be explained by methodological differences. In addition, GLP-1 is able to slow down gastric emptying. ${ }^{12}$ This could lead to increased volumes of the nutrient drink remaining in the stomach, which could also contribute to

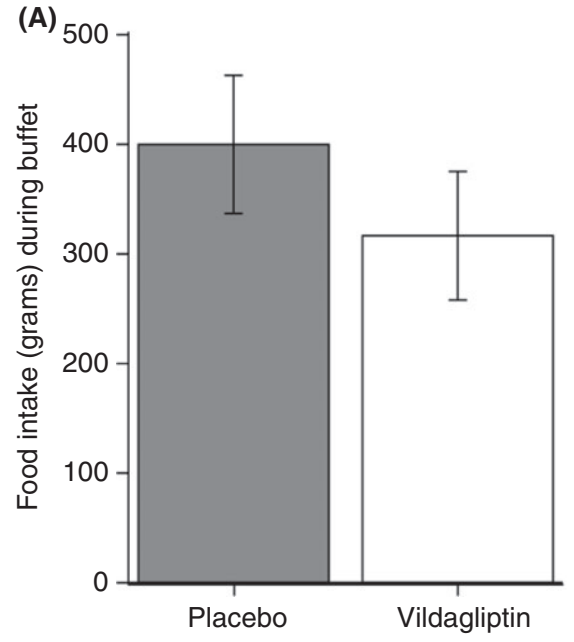

FIGURE 4 Food intake (A) and energy intake (B) during ad libitum buffet meal. A, Total food intake in weight did not differ significantly between vildagliptin and placebo $(P=0.359)$. B, There was no significant difference in total energy intake between vildagliptin and placebo $(P=0.371)$ 
increased pressures on the manometry probe and hence higher IGPs measured with high-resolution manometry. In the present protocol, gastric emptying rate was not determined.

Symptom scores were assessed during IGP measurement. No significant differences in gastrointestinal symptoms between vildagliptin and placebo were reported. Consequently, this study shows that an acute dose of vildagliptin is well-tolerated and safe.

We also investigated the effect of vildagliptin on food intake after a pre-meal load, which was administered to induce GLP-1 release and to be able to evaluate the DPP-4 inhibition effect. Our data show that, as expected, active GLP-1 levels were stable both before and after acute vildagliptin administration in the fasted state, but significantly increased after nutrient drink ingestion compared to placebo. Immediately after the pre-meal load, GLP-1 plasma concentrations were significantly higher in the vildagliptin arm compared to placebo. These findings are in concordance with previous studies in humans, showing that DPP-4 inhibition is able to enhance the postprandial concentrations of active endogenous GLP-1 or to prevent the degradation of exogenous infused GLP-1. ${ }^{16,20,24}$ Although still numerically higher, the significant difference in active GLP-1 plasma levels between vildagliptin and placebo was lost immediately before the buffet due to increased variance between the subjects. Furthermore, no differences were found in food or energy intake during the buffet between vildagliptin and placebo. Possibly, a pre-meal load with a higher concentration of glucose could have had a stronger and more sustained effect on active GLP-1 plasma levels, and consequently could have had a significant effect on food or energy intake. Alternatively, a shorter interval between pre-meal load and start of the buffet could have generated larger differences.

A first limitation of this study is that no blood sample was collected immediately after the buffet to assess changes in active GLP1 plasma concentrations after food intake. We showed that the premeal load induced a significant difference in GLP-1 plasma concentrations. Consequently, GLP-1 plasma concentrations might have been different between vildagliptin and placebo after food intake during the buffet. Another limitation of this study is the rather small sample size. The sample size of 10 participants was chosen based on a power calculation to detect differences in IGP, without taking into account differences in GLP-1 plasma concentrations or food intake. Considering the numerical (but not significant) differences in food and energy intake during the buffet and the significant difference in GLP-1 plasma levels between vildagliptin and placebo immediately after ingestion of the pre-meal load, a larger sample size could have generated significant results on these secondary outcome parameters. Alternatively, it can be suggested that a prolonged intake of vildagliptin is necessary to obtain a significant effect on food intake and to observe a longer-lasting effect on GLP-1 plasma levels.

In conclusion, the present study shows for the first time that the inhibition of DPP-4 activity in man by acute vildagliptin administration increases active GLP-1 plasma levels and inhibits gastric accommodation to a liquid meal, but had no significant effect on symptom levels and total energy or food intake. Together, these findings highlight the mechanistic importance of the role of GLP-1 in the physiological gastric response to a meal.

\section{ACKNOWLEDGEMENT}

The authors thank Joran Toth for his assistance with laboratory analysis.

Declaration of personal interests: No conflicts of interest exist for any of the authors listed above.

\section{AUTHORSHIP}

Guarantor of the article: Jan Tack.

Authors' contributions: AR, JRB and JT were involved in scientific concept and design of the study; AR, JRB and IM were involved in the acquisition of data; IM analysed the data; $A R, J R B$ and IM were involved in drafting the manuscript; ED and JT critically revised the manuscript. All authors reviewed and approved the final version of the manuscript. This research was supported by a methusalem grant assigned to JT by the University of Leuven and by the Flanders Research Foundation (FWO). AR, JRB and ED were post doctorate fellows at the University of Leuven at the time of the research conduct.

No conflicts of interest exist for any of the authors.

\section{ORCID}

Imke Masuy (iD https://orcid.org/0000-0001-9006-9287

Eveline Deloose iD https://orcid.org/0000-0001-8694-7745

Jan Tack iD https://orcid.org/0000-0002-3206-6704

\section{REFERENCES}

1. Chen T, Ajami K, McCaughan GW, Gorrell MD, Abbott CA. Dipeptidyl peptidase IV gene family. The DPIV family. Adv Exp Med Biol. 2003;524:79-86.

2. Drucker DJ, Shi Q, Crivici A, et al. Regulation of the biological activity of glucagon-like peptide 2 in vivo by dipeptidyl peptidase IV. Nat Biotechnol. 1997;15:673-677.

3. Knudsen LB, Pridal L. Glucagon-like peptide-1-(9-36) amide is a major metabolite of glucagon-like peptide-1-(7-36) amide after in vivo administration to dogs, and it acts as an antagonist on the pancreatic receptor. Eur J Pharmacol. 1996;318:429-435.

4. Fehmann HC, Habener JF. Functional receptors for the insulinotropic hormone glucagon-like peptide-I(7-37) on a somatostatin secreting cell line. FEBS Lett. 1991;279:335-340.

5. Flint A, Raben A, Astrup A, Holst JJ. Glucagon-like peptide 1 promotes satiety and suppresses energy intake in humans. J Clin Invest. 1998;101:515-520.

6. Naslund E, Gutniak M, Skogar S, Rossner S, Hellstrom PM. Glucagon-like peptide 1 increases the period of postprandial satiety and slows gastric emptying in obese men. Am J Clin Nutr. 1998;68:525530.

7. Giralt M, Vergara P. Glucagonlike peptide-1 (GLP-1) participation in ileal brake induced by intraluminal peptones in rat. Dig Dis Sci. 1999;44:322-329.

8. Tolessa T, Gutniak M, Holst JJ, Efendic S, Hellstrom PM. Inhibitory effect of glucagon-like peptide-1 on small bowel motility. Fasting but 
not fed motility inhibited via nitric oxide independently of insulin and somatostatin. J Clin Invest. 1998;102:764-774.

9. Imeryuz N, Yegen BC, Bozkurt A, Coskun T, Villanueva-Penacarrillo ML, Ulusoy NB. Glucagon-like peptide-1 inhibits gastric emptying via vagal afferent-mediated central mechanisms. Am J Physiol. 1997;273 (4 Pt 1):G920-927.

10. Delgado-Aros S, Kim DY, Burton DD, et al. Effect of GLP-1 on gastric volume, emptying, maximum volume ingested, and postprandial symptoms in humans. Am J Physiol Gastrointest Liver Physiol. 2002;282:G424-431.

11. Andrews CN, Bharucha AE, Camilleri M, et al. Effects of glucagonlike peptide-1 and sympathetic stimulation on gastric accommodation in humans. Neurogastroenterol Motil. 2007;19:716-723.

12. Janssen $P$, Rotondo A, Mule F, Tack J. Review article: a comparison of glucagon-like peptides 1 and 2. Aliment Pharmacol Ther. 2013;37:18-36.

13. Wettergren A, Maina P, Boesby S, Holst JJ. Glucagon-like peptide-1 7-36 amide and peptide YY have additive inhibitory effect on gastric acid secretion in man. Scand J Gastroenterol. 1997;32:552-555.

14. Rotondo A, Amato A, Lentini L, Baldassano S, Mule F. Glucagon-like peptide-1 relaxes gastric antrum through nitric oxide in mice. Peptides. 2011;32:60-64.

15. Bannerman DM, Rawlins J, McHugh SB, et al. Regional dissociations within the hippocampus - memory and anxiety. Neurosci Biobehav Rev 2004;28:273-283. http://www.sciencedirect.com/science/artic le/pii/S0149763404000314

16. Holst JJ, Deacon CF. Glucagon-like peptide-1 mediates the therapeutic actions of DPP-IV inhibitors. Diabetologia. 2005;48:612-615.

17. Doupis J, Veves A. DPP4 inhibitors: a new approach in diabetes treatment. Adv Ther. 2008;25:627-643.

18. Scherbaum WA, Schweizer A, Mari A, et al. Evidence that vildagliptin attenuates deterioration of glycaemic control during 2-year treatment of patients with type 2 diabetes and mild hyperglycaemia. Diabetes Obes Metab. 2008;10:1114-1124.

19. Janssen P, Verschueren S, Giao Ly H, Vos R, Van Oudenhove L, Tack J. Intragastric pressure during food intake: a physiological and minimally invasive method to assess gastric accommodation. Neurogastroenterol Motil. 2011;23:316-e154.
20. Kindt S, Tack J. Impaired gastric accommodation and its role in dyspepsia. Gut. 2006;55:1685-1691.

21. Vella A, Bock G, Giesler PD, et al. The effect of dipeptidyl peptidase-4 inhibition on gastric volume, satiation and enteroendocrine secretion in type 2 diabetes: a double-blind, placebo-controlled crossover study. Clin Endocrinol (Oxf). 2008;69:737-744.

22. He YL. Clinical pharmacokinetics and pharmacodynamics of vildagliptin. Clin Pharmacokinet. 2012;51:147-162.

23. Deloose E, Janssen P, Corsetti M, et al. Intragastric infusion of denatonium benzoate attenuates interdigestive gastric motility and hunger scores in healthy female volunteers. Am J Clin Nutr. 2017;105: 580-588.

24. Rotondo A, Janssen P, Mule F, Tack J. Effect of the GLP-1 analog liraglutide on satiation and gastric sensorimotor function during nutrient-drink ingestion. Int J Obes (Lond). 2013;37:693-698.

25. Papathanasopoulos A, Rotondo A, Janssen P, et al. Effect of acute peppermint oil administration on gastric sensorimotor function and nutrient tolerance in health. Neurogastroenterol Motil. 2013;25:e263271.

26. Delgado-Aros S, Kim D, Burton DD, et al. Effect of GLP-1 on gastric volume, emptying, maximum volume ingested, and postprandial symptoms in humans. Am J Physiol. 2002;282:G424-431.

27. Schirra J, Nicolaus $M$, Woerle HJ, Struckmeier $C$, Katschinski $M$, Goke B. GLP-1 regulates gastroduodenal motility involving cholinergic pathways. Neurogastroenterol Motil. 2009;21:609-618.e621e602.

How to cite this article: Rotondo A, Masuy I, Verbeure W, Biesiekierski JR, Deloose E, Tack J. Randomised clinical trial: the DPP-4 inhibitor, vildagliptin, inhibits gastric accommodation and increases glucagon-like peptide-1 plasma levels in healthy volunteers. Aliment Pharmacol Ther.

2019;00:1-8. https://doi.org/10.1111/apt.15195 